\title{
Throughput Optimization in Multi-Cell CDMA Networks
}

\author{
Robert Akl \\ Dept of Computer Science and Eng. \\ University of North Texas \\ Denton, Texas, 76207 \\ rakl@cse.unt.edu
}

\author{
Mort Naraghi-Pour \\ Dept of Electrical and Computer Eng. \\ Louisiana State University \\ Baton Rouge, LA 70803 \\ mort@ece.lsu.edu
}

\author{
Manju Hegde \\ Ageia Technologies, Inc. \\ Saint Louis, MO 63108 \\ mvhegde@ageia.com
}

\begin{abstract}
In this paper, we investigate the performance of a multi-cell CDMA network by determining the maximum throughput that the network can achieve for a given gradeof-service requirement, quality-of-service requirement, network topology and call arrival rate profile. Our analysis is restricted to the reverse link and accounts for mobility of users between cells. A constrained nonlinear optimization problem is formulated that maximizes the network throughput subject to upper bounds on the blocking probabilities and a lower bound on the bit energy to interference ratio. The goal is to optimize the usage of network resources, provide consistent grade-of-service for all the cells in the network, and maintain a pre-specified qualityof-service. The solution to the optimization problem yields the maximum network throughput as well as the maximum number of calls that should be admitted in each cell for a given topology and call arrival rate profile. Our optimization algorithm yields significantly higher throughput compared with traditional call admission schemes.
\end{abstract}

\section{INTRODUCTION}

Efficient use of resources is a key design problem for any cellular network, in general, and code division multiple access (CDMA) networks, in particular, where the number of simultaneous calls that can be admitted in one cell depends on the number of simultaneous calls in many cells in the network. Our goal is to maximize the throughput in the network and provide consistent grade-of-service (GoS), i.e., the call blocking rate, for all the cells in the network while at the same time maintaining the quality-of-service (QoS), i.e., the probability of loss of communication quality, for all the users.

The optimization of network throughput is a popular goal and several authors have considered this problem. In [1], the authors address the problem of jointly controlling the data rates and transmit powers of the users, so as to maximize the throughput. They formulate a classical optimization problem, modeling the constraints arising from the data rate requirements and power budgets. In [2], the authors formulate the throughput maximization problem in terms of the spreading gains and transmit powers of the users, and solve it using a nonlinear programming approach. In [3], the authors investigate the maximum throughput that can be achieved through joint rate and power adaptation in a multi-rate CDMA system. They assume conventional matched filter detection with perfect channel information and an instantaneous BER constraint. They restrict their attention to multicode or multiple processing gain schemes. In [4], the authors examine the optimization of throughput for a wideband CDMA system, where the maximum transmission power is constrained and power control may not be possible. They show that by optimally allocating bit-rate using a dynamic programming algorithm, a higher bit-rate can be delivered within the same power constraint. In [5], the authors study the throughput optimization of data traffic for a power constrained voice/data CDMA system by scheduling of data users. They found that under a given received power budget and the constraints of transmission powers, the throughput of data traffic is maximized by selecting simultaneous data users and allocating powers according to the descending order of their received power capabilities, which is defined as the product between the transmission power limit and the channel gain.

In this paper, we formulate a constrained optimization problem that maximizes the network throughput subject to upper bounds on the call blocking probabilities and a lower bound on the bit energy to interference ratio. We calculate the implied costs, which are the derivatives of the throughput function, and capture the effect of increases in the call arrival rates in one cell on the throughput of the entire network. We also take mobility of users into account and differentiate between the blocking of new calls and the blocking of handoff calls. The blocking probabilities are given by the fixed point model, which describes the blocking probabilities as a function of the total offered traffic, the total offered traffic as a function of the call arrival rates and the handoff rates, and the handoff rates as a function of the blocking probabilities and the total offered traffic. We obtain relations of the total and partial derivatives of the blocking probabilities by differentiating the fixed point relations. These are then used along with the implied costs in the solution to our throughput optimization problem.

The remainder of this paper is organized as follows. The traffic and mobility models are presented in Section II. In Section III we present the throughput optimization problem. Numerical results are presented in Section IV and finally Section V concludes the paper. The derivation of the implied costs is given in the Appendix. 


\section{TRAfFic And Mobility Model}

\section{A. Feasible States}

There does not exist among researchers a unanimous consensus on whether the CDMA system capacity is reverse or forward link limited. However, the majority of the literature published on the subject is of the former view. In light of this, in this paper we consider the reverse link capacity only. Consider a multi-cell CDMA network with spread signal bandwidth of $W$, information rate of $R$ bits/s, voice activity factor of $\alpha$, and background noise spectral density of $N_{0}$. To achieve a required bit error rate we must have $\left(\frac{E_{b}}{I_{0}}\right)_{i} \geq \Gamma$ for some constant $\Gamma$. Assuming a total of $M$ cells with $n_{i}$ calls in cell $i$, the number of calls in every cell must satisfy [6]

$$
\begin{array}{r}
n_{i}+\sum_{j=1}^{M} n_{j} \kappa_{j i} \leq \frac{W / R}{\alpha}\left(\frac{1}{\Gamma}-\frac{1}{E_{b} / N_{0}}\right)+1 \triangleq c_{e f f}, \\
\text { for } i=1, \ldots, M .
\end{array}
$$

where $\kappa_{j i}=I_{j i} / n_{j}$ is the per user inter-cell interference factor from cell $j$ to cell $i$, and where $I_{j i}$ denotes the relative average interference of cell $j$ to cell $i$.

A set of calls $\mathbf{n}=\left(n_{1}, \ldots, n_{M}\right)$ satisfying the above equations is said to be a feasible call configuration or a feasible state, i.e., one that satisfies the $\frac{E_{b}}{I_{0}}$ constraint. The right hand side of (1) is a constant which is determined by system parameters and by the desired maximum bit error rate, and can be regarded as the total number of effective channels, $c_{e f f}$, available to the system.

Denote by $\Omega$ the set of feasible states. Define the set of blocking states for cell $i$ as

$$
\mathcal{B}_{i}=\left\{\mathbf{n} \in \Omega:\left(n_{1}, \ldots, n_{i}+1, \ldots, n_{M}\right) \notin \Omega\right\} .
$$

If a new call or a handoff call arrives to cell $i$, it is blocked if the current state of the network, $\mathbf{n}$, is in $\mathcal{B}_{i}$. The call blocking probability for cell $i, B_{i}$, is the probability that $\mathbf{n} \in \mathcal{B}_{i}$.

\section{B. Mobility Model}

The call arrival process to cell $i$ is assumed to be a Poisson process with rate $\lambda_{i}$ independent of other call arrival processes. The call dwell time is a random variable with exponential distribution having mean $1 / \mu$, and it is independent of earlier arrival times, call durations and elapsed times of other users [7]. At the end of a dwell time a call may stay in the same cell, attempt a handoff to an adjacent cell, or leave the network. Let $q_{i j}$ be the probability that a call in progress in cell $i$ after completing its dwell time goes to cell $j$. If cells $i$ and $j$ are not adjacent, then $q_{i j}=0$. Define $q_{i i}$ as the probability that a call in progress in cell $i$ remains in cell $i$ after completing its dwell time. In this case a new dwell time that is independent of the previous dwell time begins immediately. We denote by $q_{i}$ the probability that a call in progress in cell $i$ departs from the network.

This mobility model is attractive because we can easily define different mobility scenarios by varying the values of these probability parameters. For example, if $q_{i}$ is constant for all $i$, then the average dwell time of a call in the network will be constant regardless of where the call originates and what the values of $q_{i i}$ and $q_{i j}$ are. Thus in this case, by varying $q_{i i}$ 's and $q_{i j}$ 's we can obtain low and high mobility scenarios and compare the effect of mobility on network attributes (e.g., throughput).

Let $\mathcal{A}_{i}$ be the set of cells adjacent to cell $i$. Let $\nu_{j i}$ be the handoff rate out of cell $j$ offered to cell $i . \nu_{j i}$ is the sum of the proportion of new calls accepted in cell $j$ that go to cell $i$ and the proportion of handoff calls accepted from cells adjacent to cell $j$ that go to cell $i$. Thus

$$
\nu_{j i}=\lambda_{j}\left(1-B_{j}\right) q_{j i}+\left(1-B_{j}\right) q_{j i} \sum_{x \in \mathcal{A}_{j}} \nu_{x j} .
$$

Equation (3) can be rewritten as

$$
\nu_{j i}=\nu\left(B_{j}, \rho_{j}, q_{j i}\right)=\left(1-B_{j}\right) q_{j i} \rho_{j},
$$

where $\rho_{j}$, the total offered traffic to cell $j$, is given by

$$
\rho_{j}=\rho\left(\mathbf{v}, \lambda_{j}, \mathcal{A}_{j}\right)=\lambda_{j}+\sum_{x \in \mathcal{A}_{j}} \nu_{x j},
$$

and where $\mathbf{v}$ denotes the matrix whose components are the handoff rates $\nu_{i j}$ for $i, j=1, \ldots M$.

The total offered traffic can be obtained from a fixed point model, which describes the offered traffic as a function of the handoff rates and new call arrival rates, the handoff rates as a function of the blocking probabilities and the offered traffic, and the blocking probabilities as a function of the offered traffic. For a given set of arrival rates, we use an iterative method to solve the fixed point equations. We define an initial value for the handoff rates. We calculate the offered traffic by adding the given values of the arrival rates to the handoff rates. The blocking probabilities are now calculated using the offered traffic. We then calculate the new values of the handoff rates and repeat.

\section{Admissible States}

A call arriving to cell $i$ is accepted if and only if the new state is a feasible state. Clearly this requires global state, i.e., the number of calls in progress in all the cells of the network. Furthermore, to compute the blocking probabilities, the probability of each state in the feasible region needs to be calculated. Since the cardinality of $\Omega$ is $O\left(c_{e f f}{ }^{M}\right)$, the calculation of the blocking probabilities has a computational complexity that is exponential in the number of cells.

In order to simplify the call admission process, we consider only those which require local state, i.e, the number of calls in progress in the current cell. To this end we define a state $\mathbf{n}$ to be admissible if

$$
n_{i} \leq N_{i} \quad \text { for } \quad i=1, \ldots, M,
$$

where $N_{i}$ is a parameter which denotes the maximum number of calls allowed to be admitted in cell $i$. Clearly the set of 
admissible states denoted $\Omega^{\prime}$ is a subset of the set of feasible states $\Omega$. The blocking probability for cell $i$ is then given by

$$
B_{i}=B\left(A_{i}, N_{i}\right)=\frac{A_{i}^{N_{i}} / N_{i} !}{\sum_{k=0}^{N_{i}} A_{i}^{k} / k !},
$$

where $A_{i}=\rho_{i} / \mu_{i}=\rho_{i} / \mu\left(1-q_{i i}\right)$. We note that the complexity to calculate the blocking probabilities in (7) is $O(M)$, and the bit error rate requirement is guaranteed since $\Omega^{\prime} \subset \Omega$.

\section{ThroughPUT OptIMIZATION}

The throughput of cell $i$ consists of two components: the new calls that are accepted in cell $i$ minus the forced termination due to handoff failure of the handoff calls into cell $i$. Hence the total throughput, $T$, of the network is

$$
\begin{aligned}
T(\mathbf{B}, \underline{\rho}, \underline{\lambda}) & =\sum_{i=1}^{M}\left\{\lambda_{i}\left(1-B_{i}\right)-B_{i}\left(\rho_{i}-\lambda_{i}\right)\right\}, \\
& =\sum_{i=1}^{M}\left\{\lambda_{i}-B_{i} \rho_{i}\right\},
\end{aligned}
$$

where $\mathbf{B}$ is the vector of blocking probabilities, $\rho$ is the vector of total offered traffic, and $\underline{\lambda}$ is the vector of call arrival rates.

We formulate a constrained nonlinear optimization problem in order to maximize the throughput subject to upper bounds on the blocking probabilities and a lower bound on the signalto-interference constraints in (1). The goal is to optimize the usage of network resources and provide consistent grade-ofservice (GoS), i.e., the call blocking rate, for all the cells in the network while at the same time maintaining the quality-ofservice (QoS), i.e., the probability of loss of communication quality, for all the users. In the optimization problem the arrival rates and the maximum number of calls that are allowed to be admitted in the cells are the independent variables. This is given in the following

$$
\begin{aligned}
\max _{\left(\lambda_{1}, \ldots, \lambda_{M}\right),\left(N_{1}, \ldots, N_{M}\right)} & T(\mathbf{B}, \underline{\rho}, \underline{\lambda}), \\
\text { subject to } & B\left(A_{i}, N_{i}\right) \leq \eta, \\
& N_{i}+\sum_{j=1}^{M} N_{j} \kappa_{j i} \leq c_{e f f}, \\
& \text { for } \quad i=1, \ldots, M,
\end{aligned}
$$

The optimization problem in (9) is a mixed integer programming (MIP) problem. One technique to solve the MIP problem is based on dividing the problem into a number of smaller problems in a method called branch and bound [8]. Branch and bound is a systematic method for implicitly enumerating all possible combinations of the integer variables in a model. The number of subproblems and branches required can become extremely large.

By relaxing the integer variables $N_{i}, i=1, \ldots, M$, to continuous variables, the optimization in (9) is solved using a Sequential Quadratic Programming (SQP) method [9]. In this method, a Quadratic Programming subproblem is solved at each iteration. A solution to the fixed point equations is calculated iteratively. An estimate of the Hessian of the Lagrangian is updated at each iteration using the BroydenFletcher-Goldfarb-Shanno (BFGS) formula [10]. A line search is performed using a merit function. The Quadratic Programming subproblem is solved using an active set strategy.

In order to use the SQP method, we need to evaluate the derivatives of $T$ with respect to $\mathbf{N}$ and $\underline{\lambda} . T(\mathbf{B}, \underline{\rho}, \underline{\lambda})$ is an implicit function of $\mathbf{N}=\left(N_{1}, \ldots, N_{M}\right)$ and $\underline{\lambda}$. We can obtain relations of total and partial derivatives of the throughput by differentiating the fixed point equations. These relations are manipulated to obtain a system of linear equations in the derivatives of the offered traffic with respect to the number of calls admitted and the arrival rates. In [11], we calculate the implied cost, i.e., the derivative of $T$ with respect to the implicit variable $\mathbf{N}$. The calculation of the implied cost of $T$ with respect to the new-call arrival rates is given in the Appendix.

\section{Numerical Results}

The following results have been obtained for the twentyseven cell CDMA network shown in Fig. 1. The base stations are located at the centers of a hexagonal grid whose radius is 1732 meters. Base station 1 is located at the center. The base stations are numbered consecutively in a spiral pattern. The COST-231 propagation model with a carrier frequency of 1800 $\mathrm{MHz}$, average base station height of 30 meters, and average mobile height of 1.5 meters is used to determine the coverage region. We assume the following for the analysis. The path loss coefficient is 4 . The shadow fading standard deviation is $6 \mathrm{~dB}$. The processing gain is $21.1 \mathrm{~dB}$. The bit energy to interference ratio threshold, $\Gamma$, is $9.2 \mathrm{~dB}$. The interference to background noise ratio is $10 \mathrm{~dB}$. The voice activity factor is 0.375 . For more details on the choice of these parameters refer to [12]. Per user inter-cell interference factors are evaluated numerically by dividing the whole area into small grids of size $150 \mathrm{~m}$ by $150 \mathrm{~m}$ (for more detail see [6]). The blocking probability threshold, $\eta$, is set to 0.02 .

We consider three mobility scenarios: no mobility, low mobility, and high mobility of users. The following probabilities are chosen for the no mobility case: $q_{i j}=0, q_{i i}=0.3$ and $q_{i}=0.7$ for all cells $i$ and $j$. For the low and high mobility case, the mobility probability parameters are given in Tables I and II, respectively. In all three cases, the probability that a call leaves the network after completing its dwell time is 0.7. Thus, the average dwell time of a call in the network is constant regardless of where the call originates and the mobility scenario used.

In the following, we compare our results to a call admission control algorithm where the maximum number of calls that can be admitted in each cell is the same, i.e., $N_{1}=N_{2}=$ $\ldots=N_{M}=N$ (irrespective of the call arrival rate profile in the network). We also optimize the revenue for this algorithm subject to a lower bound on the bit energy to interference ratio. In the sequel, the optimized algorithm is referred to as a traditional call admission control (CAC) algorithm. Note that 
TABLE I

THE LOW MOBILITY PROBABILITIES.

\begin{tabular}{|c||c|c|c|}
\hline$\left\|\mathcal{A}_{i}\right\|$ & $q_{i j}$ & $q_{i i}$ & $q_{i}$ \\
\hline \hline 3 & 0.020 & 0.240 & 0.700 \\
\hline 4 & 0.015 & 0.240 & 0.700 \\
\hline 5 & 0.012 & 0.240 & 0.700 \\
\hline 6 & 0.010 & 0.240 & 0.700 \\
\hline \hline
\end{tabular}

TABLE II

THE HIGH MOBILITY PROBABILITIES.

\begin{tabular}{|c||c|c|c|}
\hline$\left\|\mathcal{A}_{i}\right\|$ & $q_{i j}$ & $q_{i i}$ & $q_{i}$ \\
\hline \hline 3 & 0.100 & 0.000 & 0.700 \\
\hline 4 & 0.075 & 0.000 & 0.700 \\
\hline 5 & 0.060 & 0.000 & 0.700 \\
\hline 6 & 0.050 & 0.000 & 0.700 \\
\hline
\end{tabular}

- $\left\|\mathcal{A}_{i}\right\|$ is the number of cells adjacent to cell $i$.

- $q_{i j}$ is the probability a call in cell $i$ goes to cell $j$.

- $q_{i i}$ is the probability a call in cell $i$ stays in cell $i$.

- $q_{i}$ is the probability a call in cell $i$ leaves the network.

this algorithm will be optimal in the sense of (9) in the case of equal call arrival rates, equal mobility probabilities for all the cells, and a network with a large number of cells (in which edge effects can be ignored).

We choose the call arrival rates to be equal to $\lambda$ calls per unit time for all cells except those in Group A (i.e., cells 5, 13, 14, and 23) and Group B (i.e., cells 2, 8, 9, and 19) as shown in Fig. 1. For Groups A and B the call arrival rates are equal to $5 \lambda$ calls per unit time. The total offered traffic per cell (the sum of the call arrival rate and the handoff rate) is shown in brackets for the no mobility, low mobility, and high mobility cases in Figures 1, 2, and 3, respectively. The maximum number of calls that can be admitted in each cell, calculated from equation (9), is shown in parentheses in the same figures. For the traditional CAC algorithm with the same blocking probability threshold, system parameters, and the bit energy to interference ratio requirement, the maximum number of calls that could be admitted in each cell would be 18 . In our algorithm for the no mobility case, it can be seen that for the cells belonging to Groups A and B the maximum number of calls admitted has increased from 18 to 22-24, while for all other cells it has decreased from 18 to 7-9. It can be seen that our algorithm trades off the calls in the cells with low arrival rate for the calls in the cells with high arrival rate. As the mobility model changes from no mobility to high mobility, the handoff rates increase thus increasing the total offered traffic per cell. For the high mobility case (Fig. 3), the maximum number of calls admitted now ranges from 20 to 23 for cells belonging to Groups $\mathrm{A}$ and $\mathrm{B}$, and from 7 to 11 for all other cells.

The throughput of each cell resulting from our algorithm and the traditional CAC algorithm for the no mobility, low mobility, and high mobility cases are given in Figures 4, 5, and 6 , respectively. In these figures, the circle and star at the two ends of a vertical bar indicates the throughput of

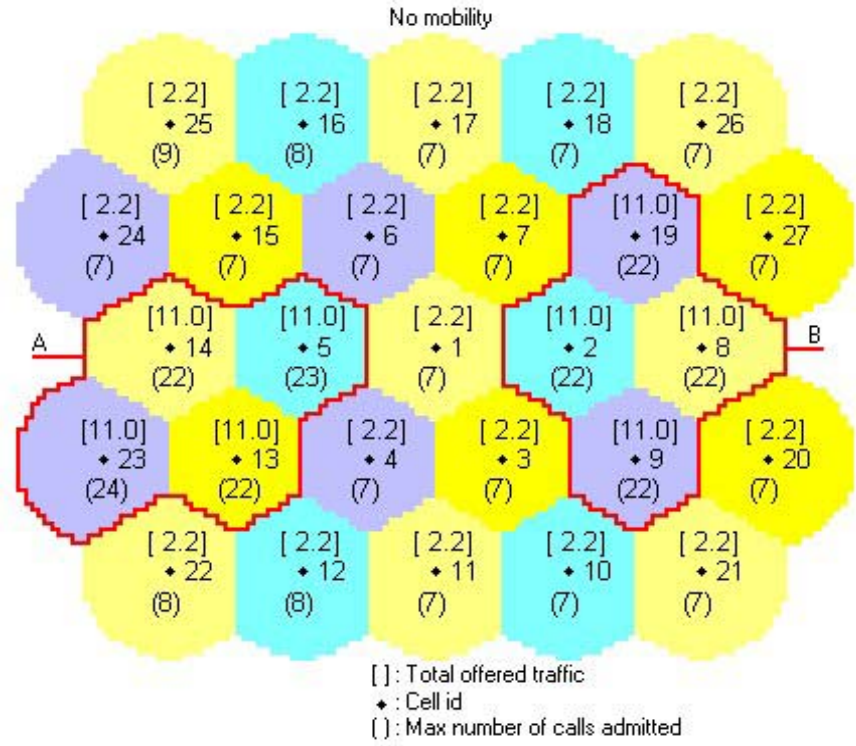

Fig. 1. Total offered traffic and maximum number of calls allowed to be admitted per cell for the twenty-seven cell CDMA network with no mobility of users.

the traditional CAC algorithm and our algorithm, respectively, for the cell whose id is shown on the horizontal axis. The traditional CAC algorithm has a total network throughput equal to $96.03,99.08$, and 102.43 calls per unit time for the no mobility, low mobility, and high mobility cases, respectively. Our optimization increases the throughput for the network to 127.02, 131.40, and 136.52 calls per unit time for the no mobility, low mobility, and high mobility cases, respectively, which is a $32 \%$ increase in throughput over the traditional CAC algorithm for the same guaranteed blocking probability threshold of 0.02. The value of $\lambda$ calculated from (9) increases from 1.65 (in the traditional CAC algorithm) to 2.19 calls per unit time (in our optimization) for the no mobility case, from 1.70 to 2.27 calls per unit time for the low mobility case, and from 1.75 to 2.37 calls per unit time for the high mobility case. Due to its call trade-offs between low and high traffic cells, this algorithm is able to better accommodate the unequal call arrival rates in the network and achieve higher throughput in all the cells for the same guaranteed GoS.

Fig. 7 presents the values of the throughput for the entire network for both our optimization and the traditional CAC algorithm as the blocking probability threshold is varied from 0.01 to 0.1 . Fig. 7 clearly demonstrates the significant increase in network throughput as a result of using our optimization versus the traditional algorithm.

\section{CONCLUSIONS}

We have investigated the network performance by determining the throughput that the network can achieve for a given network topology and call arrival rate profile. We formulated a constrained optimization problem that maximizes the network throughput subject to upper bounds on the blocking probabilities and a lower bound on the bit energy to interference 


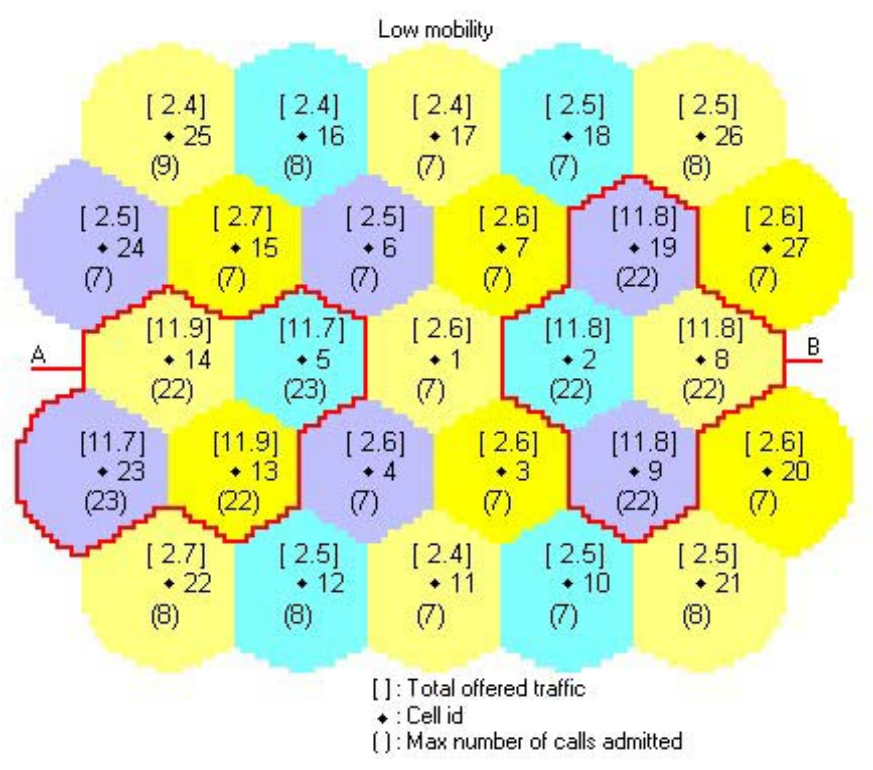

Fig. 2. Total offered traffic and maximum number of calls allowed to be admitted per cell for the twenty-seven cell CDMA network with low mobility of users.

ratio. The blocking probabilities are given by the fixed point model. We obtained relations of the total and partial derivatives of the blocking probabilities by differentiating the fixed point relations. They are used in the solution to the optimization problem which yields the maximum network throughput as well as the maximum number of calls that should be admitted in each cell for the network to guarantee a given gradeof-service and quality-of-service requirements. For unequal call arrival rates, our optimization algorithm achieved a $32 \%$ increase in throughput over the traditional CAC algorithm.

\section{APPENDIX}

\section{Calculation of the Implied Cost w.r.t. $\underline{\lambda}$}

In what follows we determine the implied cost of the throughput with respect to the new-call arrival rates. The total derivative of the throughput function with respect to a new-call arrival rate is given by

$$
\begin{aligned}
\frac{d T(\mathbf{B}, \underline{\rho}, \underline{\lambda})}{d \lambda_{k}}= & 1+\sum_{i=1}^{M}\left\{\frac{\partial T(\mathbf{B}, \underline{\rho}, \underline{\lambda})}{\partial B_{i}} \frac{d B\left(A_{i}, N_{i}\right)}{d \lambda_{k}}\right. \\
& \left.+\frac{\partial T(\mathbf{B}, \underline{\rho}, \underline{\lambda})}{\partial \rho_{i}} \frac{d \rho\left(\mathbf{v}, \lambda_{i}, \mathcal{A}_{i}\right)}{d \lambda_{k}}\right\}
\end{aligned}
$$

The partial derivatives needed in (10) are

$$
\frac{\partial T(\mathbf{B}, \underline{\rho}, \underline{\lambda})}{\partial B_{i}}=-\rho_{i}
$$

and

$$
\frac{\partial T(\mathbf{B}, \underline{\rho}, \underline{\lambda})}{\partial \rho_{i}}=-B_{i}
$$

From (7) we get

$$
\frac{d B\left(A_{i}, N_{i}\right)}{d \lambda_{k}}=\frac{\partial B\left(A_{i}, N_{i}\right)}{\partial A_{i}} \frac{\partial A_{i}}{\partial \rho_{i}} \frac{d \rho\left(\mathbf{v}, \lambda_{i}, \mathcal{A}_{i}\right)}{d \lambda_{k}} .
$$

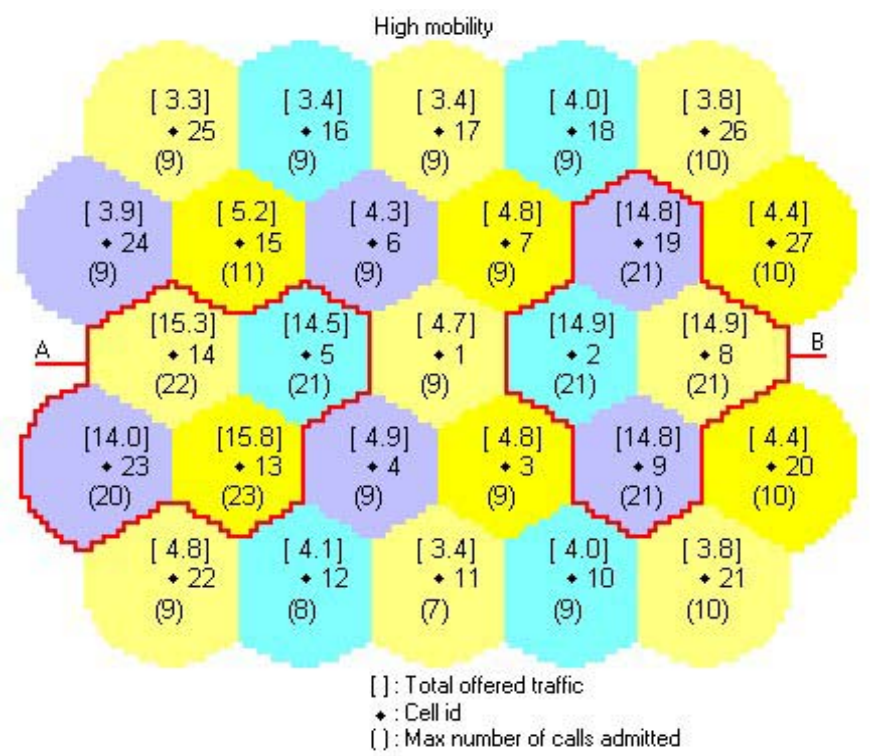

Fig. 3. Total offered traffic and maximum number of calls allowed to be admitted per cell for the twenty-seven cell CDMA network with high mobility of users.

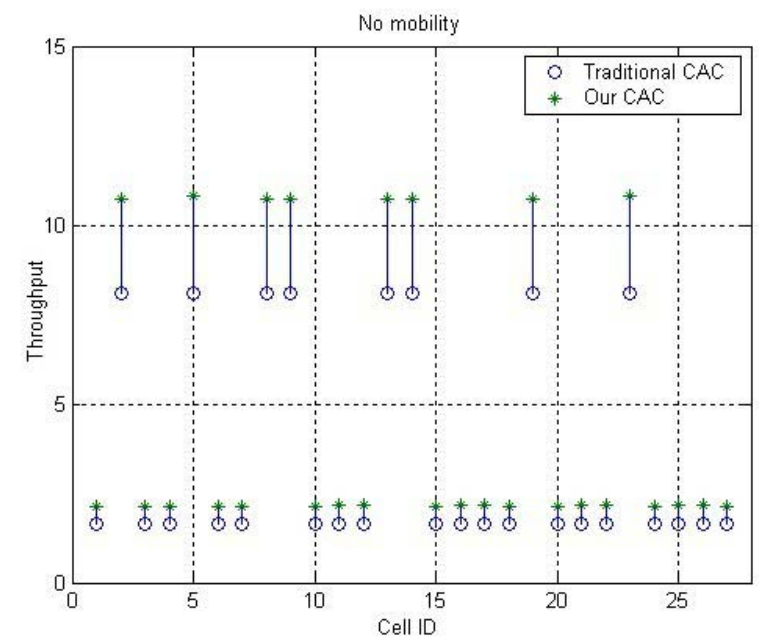

Fig. 4. Maximum throughput in every cell for the network in Fig. 1.

The total derivative needed in (13) and (10) can be obtained from (5) as follows

$$
\begin{aligned}
\frac{d \rho\left(\mathbf{v}, \lambda_{i}, \mathcal{A}_{i}\right)}{d \lambda_{k}}= & \sum_{x \in \mathcal{A}_{i}} \frac{\partial \rho\left(\mathbf{v}, \lambda_{i}, \mathcal{A}_{i}\right)}{\partial \nu_{x i}} \frac{d \nu\left(B_{x}, \rho_{x}, q_{x i}\right)}{d \lambda_{k}} \\
& +\frac{\partial \rho\left(\mathbf{v}, \lambda_{i}, \mathcal{A}_{i}\right)}{\partial \lambda_{k}}
\end{aligned}
$$

where $\frac{\partial \rho\left(\mathbf{v}, \lambda_{i}, \mathcal{A}_{i}\right)}{\partial \lambda_{k}}=\mathcal{I}_{\{i=k\}}$. From (5) we get $\frac{\partial \rho\left(\mathbf{v}, \lambda_{i}, \mathcal{A}_{i}\right)}{\partial \nu_{x i}}=$ $\mathcal{I}_{\left\{x \in \mathcal{A}_{i}\right\}}$. Finally, from (4), the derivative of the handoff rate with respect to the call arrival rate is given by

$$
\begin{aligned}
\frac{d \nu\left(B_{x}, \rho_{x}, q_{x i}\right)}{d \lambda_{k}}= & \frac{\partial \nu\left(B_{x}, \rho_{x}, q_{x i}\right)}{\partial B_{x}} \frac{d B\left(A_{x}, N_{x}\right)}{d \lambda_{k}} \\
& +\frac{\partial \nu\left(B_{x}, \rho_{x}, q_{x i}\right)}{\partial \rho_{x}} \frac{d \rho\left(\mathbf{v}, \lambda_{x}, \mathcal{A}_{x}\right)}{d \lambda_{k}}
\end{aligned}
$$




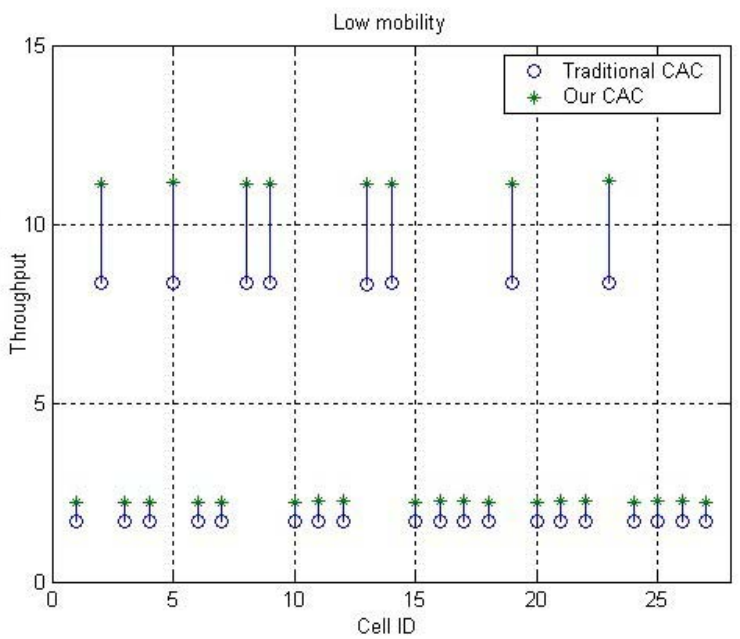

Fig. 5. Maximum throughput in every cell for the network in Fig. 2.

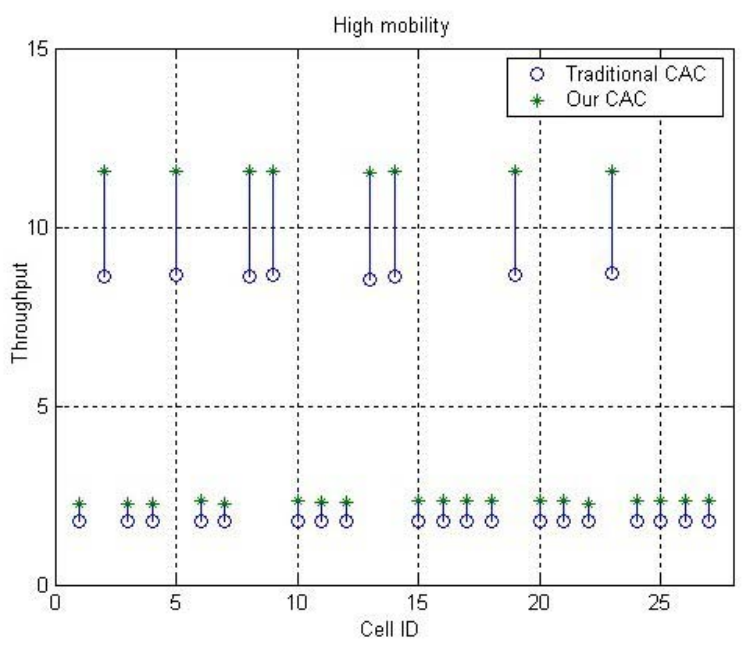

Fig. 6. Maximum throughput in every cell for the network in Fig. 3.

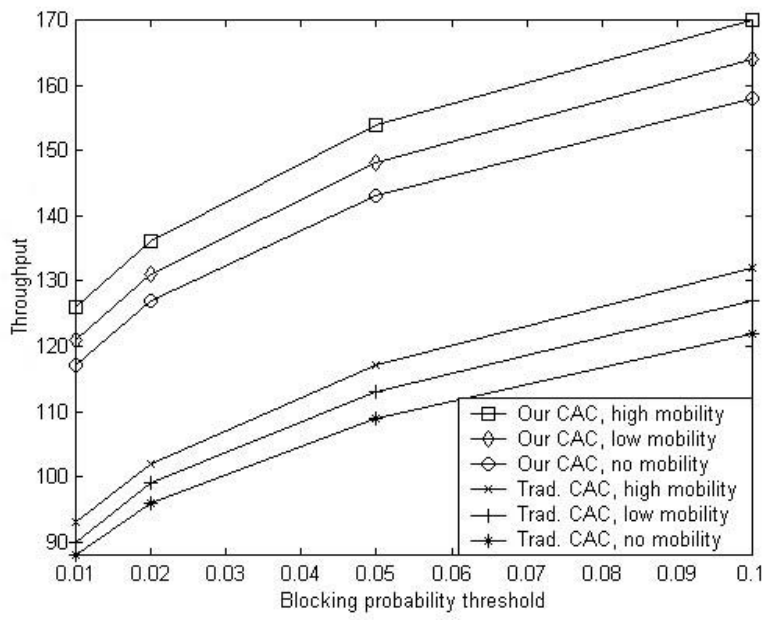

Fig. 7. Maximum throughput in the network for different values of the blocking probability threshold.
The partial derivatives needed in (15) are obtained as follows

$$
\begin{gathered}
\frac{\partial \nu\left(B_{x}, \rho_{x}, q_{x i}\right)}{\partial B_{x}}=-q_{x i} \rho_{x}, \\
\frac{\partial \nu\left(B_{x}, \rho_{x}, q_{x i}\right)}{\partial \rho_{x}}=\left(1-B_{x}\right) q_{x i} .
\end{gathered}
$$

Equations (16) and (17) are substituted back in (15) which is substituted in (14) which results in expressions of the total derivatives, $\frac{d \rho\left(\mathbf{v}, \lambda_{i}, \mathcal{A}_{i}\right)}{d \lambda_{k}}$, in terms of $\frac{d B\left(A_{x}, N_{x}\right)}{d \lambda_{k}}$ and $\frac{d \rho\left(\mathbf{v}, \lambda_{x}, \mathcal{A}_{x}\right)}{d \lambda_{k}}$. Then (13) is substituted in (14) which results in expressions of the total derivatives, $\frac{d \rho\left(\mathbf{v}, \lambda_{i}, \mathcal{A}_{i}\right)}{d \lambda_{k}}$, in terms of $\frac{d \rho\left(\mathbf{v}, \lambda_{x}, \mathcal{A}_{x}\right)}{d \lambda_{k}}$. The set of simultaneous linear equations can be solved and the results substituted back in (10) along with equations (11) and (12). This completes the derivation of the implied cost and the values of the derivatives of the throughput with respect to the call arrival rates. Implied costs capture the effect of increases in the call arrival rate in one cell on the throughput of the entire network.

\section{REFERENCES}

[1] D. Ayyagari and A. Ephremides, "Power control based admission algorithms for maximizing throughput in DS-CDMA networks with multimedia traffic," IEEE Wireless Commun. and Networking Conf., vol. 2, pp. 631-635, September 1999.

[2] S. Ulukus and L. Greenstein, "Throughput maximization in CDMA uplinks using adaptive spreading and power control," IEEE Sixth International Symposium on Spread Spectrum Techniques and Applications, vol. 2, pp. 565-569, September 2000.

[3] S. Jafar and A. Goldsmith, "Adaptive multicode CDMA for uplink throughput maximization," IEEE Veh. Technol. Conf., vol. 1, pp. 546550, May 2001.

[4] S. Kahn, M. Gurcan, and O. Oyefuga, "Downlink throughput optimization for wideband CDMA systems," IEEE Commun. Lett., vol. 7, no. 5, pp. 251-253, May 2003.

[5] T. Shu and Z. Niu, "A channel-adaptive and throughput-efficient scheduling scheme in voice/data DS-CDMA networks with constrained transmission power," IEEE International Conf. on Commun., vol. 3, pp. 2229-2233, May 2003.

[6] R. Akl, M. Hegde, M. Naraghi-Pour, and P. Min, "Multi-cell CDMA network design," IEEE Trans. Veh. Technol., vol. 50, no. 3, pp. 711722, May 2001.

[7] E. D. Re, R. Fantacci, and G. Giambene, "Handover and dynamic channel allocation techniques in mobile cellular networks," IEEE Trans. Veh. Technol., vol. 44, no. 2, pp. 229-237, 1995.

[8] G. Nemhauser and L. Wolsey, Integer and Combinatorial Optimization. Wiley, 1988.

[9] M. Powell, "A fast algorithm for nonlinearly constrained optimization calculations," Numerical Analysis, vol. 630, 1978.

[10] T. Coleman and Y. Li, "An interior, trust region approach for nonlinear minimization subject to bounds," SIAM Journal on Optimization, vol. 6 , pp. 418-445, 1996.

[11] R. Akl, M. Hegde, M. Naraghi-Pour, and P. Min, "Call admission control scheme for arbitrary traffic distribution in CDMA cellular systems," IEEE Wireless Commun. and Networking Conf., pp. 465-470, September 2000.

[12] A. Viterbi, CDMA Principles of Spread Spectrum Communication. Addison-Wesley, 1995. 